\title{
Wireless and cuffless monitoring of blood pressure in the operating room: the role of optical beat-to-beat technology
}

\section{Yan Degiorgis ${ }^{1}$, Josep Solà ${ }^{2}$, Fabian Braun², Nicolas Pierrel ${ }^{1}$, Christophe Verjus ${ }^{2}$, Mattia Bertschi $^{2}$, Patrick Schoettker ${ }^{1}$}

1. Centre Hospitalier Universitaire Vaudois (CHUV)

2. Centre Suisse d'Electroinique et Microtechnique (CSEM)

\section{Background and Goal of Study:}

The aim of this study is to demonstrate feasibility and efficacy of tracking beat-by-beat changes of blood pressure during induction of general anesthesia by simple optical means in comparison with an invasive arterial signal.

\section{Materials and Methods:}

The study included 40 patients necessitating invasive arterial blood pressure monitoring for general anesthesia. Anesthesia was induced after arterial catheter had been inserted under local anesthesia, while photo-plethysmographic signals were acquired at the fingertip.

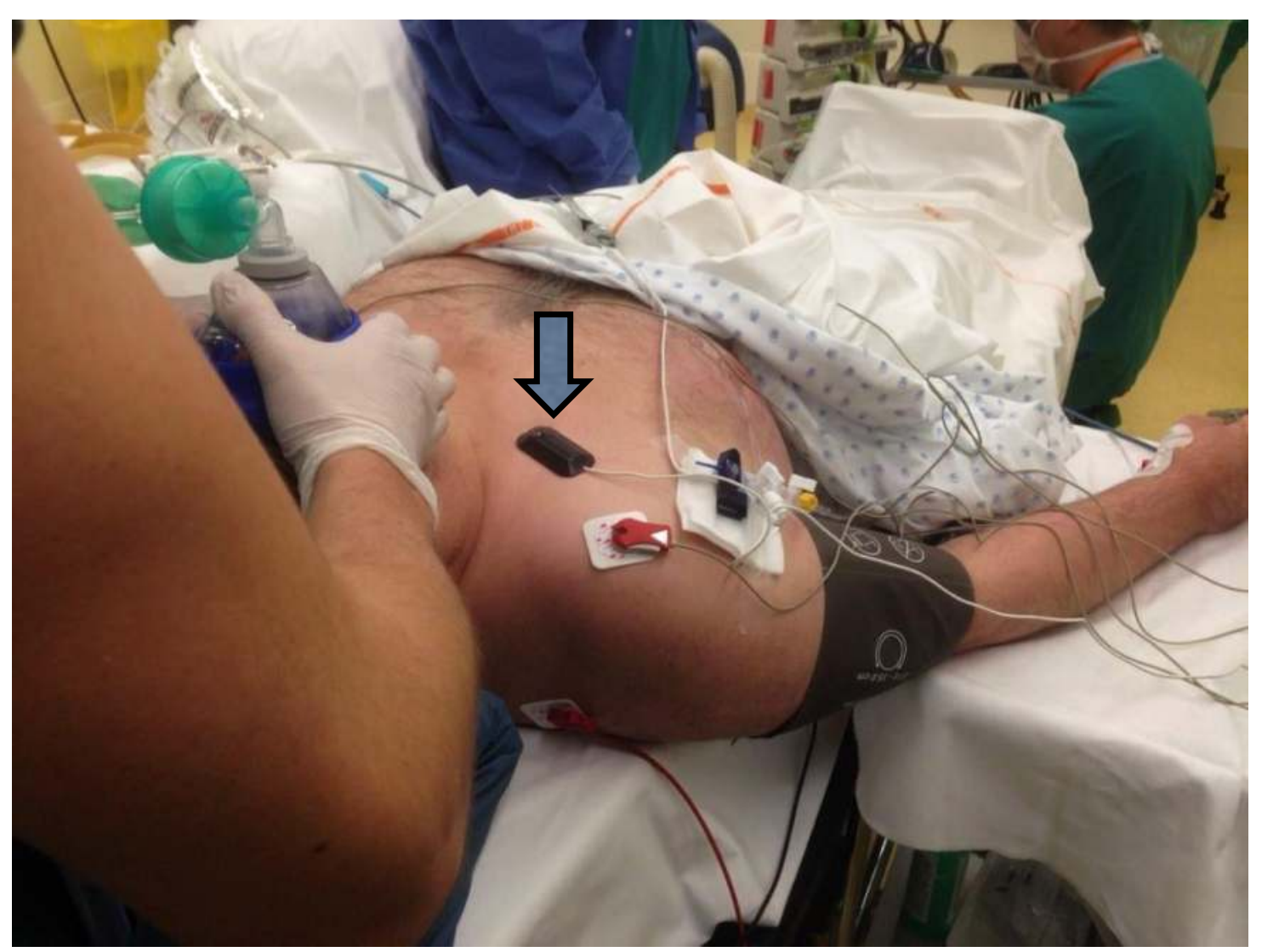

A dedicated Pulse Wave Analysis (PWA) algorithm continuously generated blood pressure estimates that were further compared with reference readings provided by the arterial catheter.

\section{Results and Discussion:}

Figures 1 illustrates the results of cuffless systolic blood pressure estimations compared to invasive arterial blood pressure references. Each data point corresponds to a patient's heart beat. First row of each figure depicts correlation plots between invasive systolic blood pressure values and respectively, heart rate estimates (left plot) and cuffless blood pressure estimates (right plot).
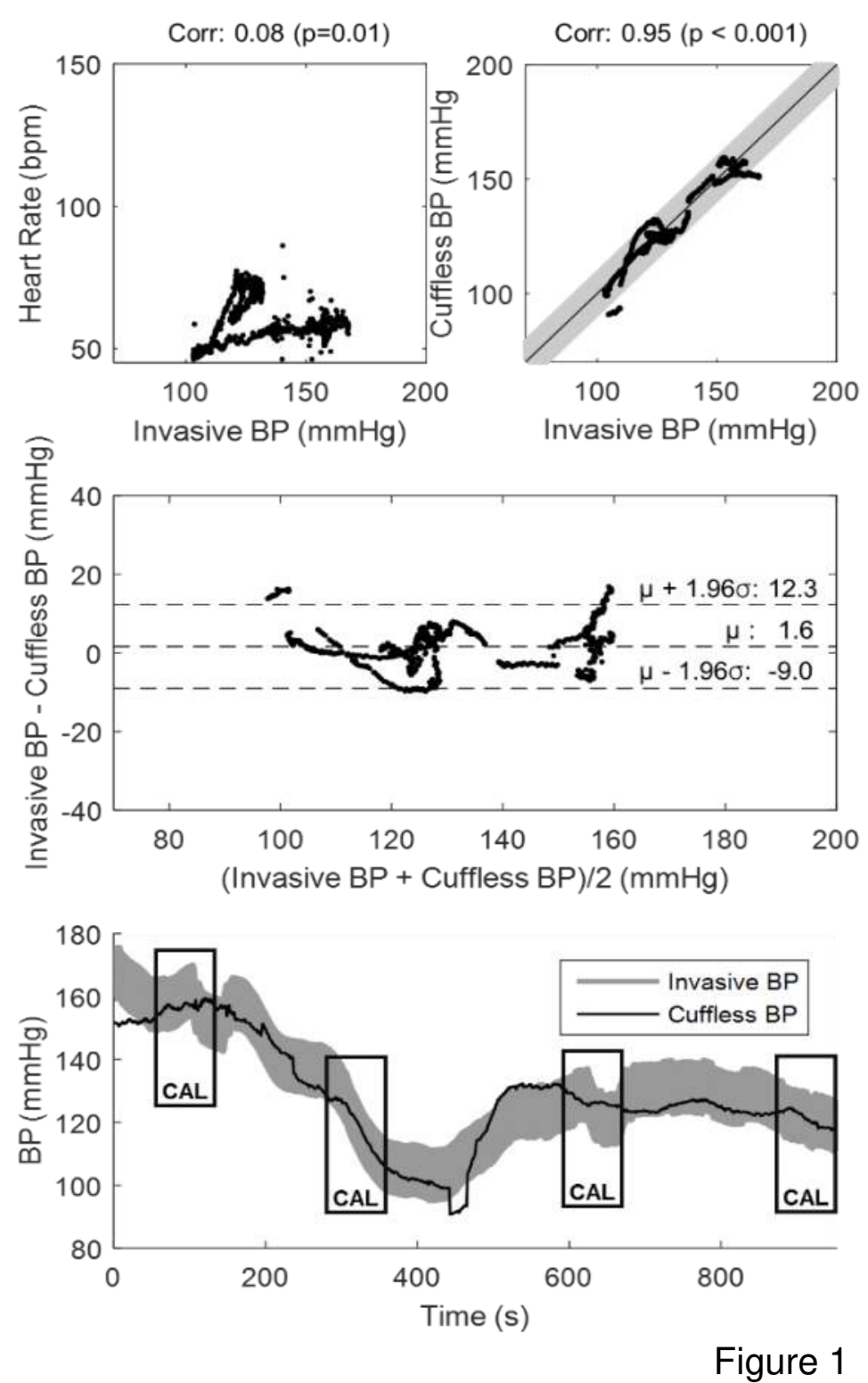

Conclusion(s):

Estimating patients' blood pressure changes in relation to induction of general anesthesia by means of a simple optical probe at the fingertip associated to a specific pulse-wave analysis is feasible and reliable. 